allows for directed clinical therapy and possible resolution of the disease.

\section{FOLLICULAR DENDRITIC CELL SARCOMA OF THE NASOPHARYNX}

K. Middleton, S. Wong, A. Parker

St Vincent's Hospital, Sydney, Australia

Background: Follicular dendritic cell sarcoma (FDCS) is a rare low-grade sarcoma, which presents as intranodal or, more commonly, extranodal disease at a wide variety of sites. To date, only eight cases of FDCS of the nasopharynx are reported. ${ }^{1,2}$ The morphological spectrum includes fascicular, whorled and sheetlike architecture with bland spindled to ovoid cells and possible nuclear pseudoinclusions. Occasionally, high-grade features including cytological atypia, numerous mitoses and necrosis are seen.

Aims: To present the case of a 24-year-old female with a nasopharyngeal mass, subsequently diagnosed as FDCS.

Methods: A biopsy was obtained which showed sheets of malignant cells, admixed with lymphocytes and occasional neutrophils. The cells had pale fibrillary cytoplasm, appearing syncytial in some areas. The nuclei were ovoid with fine chromatin, although with significant anisonucleosis, frequent large nuclear pseudoinclusions and occasional nucleoli. Numerous mitoses and areas of necrosis were present. Despite this unusual morphology, immunohistochemistry was positive for CD21, CD23 and CD35, indicative of FDCS.

Results and conclusions: FDCS is an uncommon entity which, combined with variant morphology and an unusual site, can lead to a high risk of misdiagnosis; reported in four of six nasopharyngeal cases $(67 \%){ }^{2}$ A high index of suspicion and broad immunohistochemical panel are required for diagnosis, particularly when high-grade features are present

\section{References}

1. Karabulut B, Orhan KS, Guldiken Y, et al. Follicular dendritic cell sarcoma of the nasopharynx. Int J Oral Maxillofac Surg 2012; 41: 218-20.

2. Duan G, Wu F, Zhu J, et al. Extranodal follicular dendritic cell sarcoma of the pharyngeal region: A potential diagnostic pitfall, with literature review. Am J Clin Pathol 2010; 133: 49-58.

\section{CILIATED MUCINOUS EPITHELIUM IS NOT ENTIRELY INNOCENT! RARE VARIANT OF MUCOEPIDERMOID CARCINOMA: A CASE REPORT}

Z. Househ $^{1}$, J. McGuinness ${ }^{2}$, K. Tran ${ }^{1}$

${ }^{1}$ Department of Anatomical Pathology, SEALS-NSW Health Pathology, Kogarah, NSW, Australia; and ${ }^{2}$ Head and Neck Surgical Department, St George Hospital, Kogarah, NSW, Australia

Background: Mucoepidermoid carcinoma (MEC) is the most common malignant salivary gland tumour. The classical form of MEC has three types of cells; epidermoid, intermediate and clear/ mucinous. Fusions involving MAML2 gene are thought to be specific for MEC and have been used as an adjunct tool to confirm diagnosis of MEC.

Aims: We present a case of an unusual histologic variant of MEC of the salivary gland in which MAML2 gene alteration detection was needed to establish the diagnosis.
Methods: A 63-year-old man presented with a slow growing, painless right parotid swelling for 9 years. He underwent a right superficial parotidectomy. Macroscopically, there was a well demarcated $4 \mathrm{~cm}$ multilocular cystic lesion filled with mucinous material.

Results and conclusion: Microscopic examination confirmed an encapsulated multiloculated cystic tumour filled with mucin which were separated by thick fibrous septa. The cysts were lined by a single layer of low-grade focally ciliated mucinous epithelium. There was no squamous differentiation or intermediate cells. Histological differential diagnoses were mucinous cystadenoma and developmental ciliated cyst. For completion a tumour block was sent for fluorescence in situ hybridisation (FISH) testing which confirmed MAML2 gene rearrangement. The lesion was therefore diagnosed as a mucinous ciliated variant of low-grade MEC.

The ciliated variant of MEC is extremely rare with only two reported cases in the literature. However, the total absence of epidermoid or intermediate components has not been reported This case highlights the importance of utilising MAML2 fusion testing on salivary gland lesions with mucinous components to detect unusual variants of MEC.

\section{UNDERSTANDING THE CLINICAL UTILITY AND PROGNOSTIC IMPLICATIONS OF THE NEW AJCC8 STAGING SYSTEM FOR CUTANEOUS SQUAMOUS CELL CARCINOMA: A SURVEY OF HEAD AND NECK CANCER SPECIALISTS}

F. Watts $^{1}$, J. Clark ${ }^{2,3,4}$, R. Gupta ${ }^{1,2,3}$

${ }^{1}$ Department of Tissue Pathology and Diagnostic Oncology, Royal Prince Alfred Hospital, Sydney, Australia; ${ }^{2}$ Sydney Head and Neck Cancer Institute, Chris O'Brien Lifehouse, Sydney, Australia; ${ }^{3}$ South West Clinical School, University of NSW, Australia; and ${ }^{4}$ Central Clinical School, University of Sydney, Sydney, Australia

Background: In order to have clinical relevance and assist in treatment decisions, a cancer staging system must stratify patients into prognostically meaningful groups; outcomes should be homogenous within groups, but distinct between them (AJCC8, 2017).

The staging system for head and neck cutaneous squamous cell carcinoma (HNcSCC) in the AJCC 8, has been criticised for its lack of homogeneity and distinctiveness, with no difference in survival between pT2 and pT3 tumours, nor between stage III and stage IV disease. The nodal staging system is likewise a poor predictor of patient outcomes.

In addition, the AJCC8 staging system for cutaneous SCC is similar to that of mucosal SCC, despite the vastly different biological behaviour of these diseases.

Aim: To understand the experience of head and neck cancer surgeons, pathologists, radiation and medical oncologists in using the AJCC8 in routine management of $\mathrm{HNcSCC}$ patients. Method: A literature review was undertaken to: (1) determine the prognostic utility of HNcSCC AJCC8 staging system, (2) identify tumour factors which may influence the staging system, and (3) identify avenues to improve the staging system. An eighteen-part online questionnaire was administered to surgeons, surgical pathologists, oncologists, and radiation oncologists, designed to elicit their views on the prognostic utility of this staging system. 